\section{MS9-P3 Investigating nanocrystalline drugs embedded in a polymeric matrix by Debye Function Analysis}

Carlotta Giacobbe ${ }^{1}$, Dritan Hasa ${ }^{2}$, Dario Voinovich ${ }^{2}$, Antonio Cervellino $^{3}$, Norberto Masciocchi ${ }^{4}$, Antonietta Guagliardi ${ }^{5}$

1. European Synchrotron Radiation Facility CS 40220 - 38043 Grenoble Cedex 9, France

2. Department of Pharmaceutical Sciences, University of Trieste, P. le Europa 1, I-34127 Trieste, Italy

3. Paul Scherrer Institut, 5232 Villigen PSI, Switzerland

4. Dipartimento di Scienza e Alta Tecnologia, Università dell'Insubria, I-22100 Como, Italy

5. Istituto di Cristallografia, CNR, I-22100 Como, Italy

\section{email: giacobbe@esrf.fr}

The release rate of a solid drug is directly related to particle size; smaller crystals have, in fact, an enhanced ability to reach their physiological target ${ }^{[1]}$. To this goal, the use of mechanical energy, inducing size/morphological modifications, represents a straightforward, green, and innovative approach. The process takes the name of mechano-chemical activation, and has recently been introduced as an efficient pharmaceutical processing technique. ${ }^{[2]}$ The release rate of a solid drug is directly related to particle size; smaller crystals have, in fact, an enhanced ability to reach their physiological target ${ }^{[1]}$. To this goal, the use of mechanical energy, inducing size/morphological modifications, represents a straightforward, green, and innovative approach. The process takes the name of mechanochemical activation, and has recently been introduced as an efficient pharmaceutical processing technique. ${ }^{[2]}$ In our work, coground mixtures of Vinpocetine (VIN) $\left(\mathrm{C}_{22} \mathrm{H}_{26} \mathrm{~N}_{2} \mathrm{O}_{2}\right)$, a poorly soluble drug used for the treatment of cognitive disorders and related symptoms ${ }^{[3]}$, and Cross-Linked Polyvinylpyrrolidone ( $\left.\mathrm{C}_{\mathrm{H}} \mathrm{NO}\right) \mathrm{N}$ (PVP-CL), have been investigated at variable milling time and 1:4 and 1:7 VIN:PVP weight ratios. The Debye Function Analysis (DFA) of several mixtures is applied for the first time to extract information about structure, size and size distribution, morphology and amorphization of drug nanoparticles. To this aim, we used high resolution data collected at the Material Science beamline MS-X04SA of the Swiss Light Source, and the DEBUSSY suite of programs ${ }^{[4]}$ modeling the total (Bragg and diffuse) sample scattering. Quantitative results on the diverse microstructure modifications controlled by the milling time and the drug-to-polymer ratio will be presented. They show a clear trend between time, size distribution and weight ratio. Moreover, investigations on the biopharmaceutical performance of the most activated systems are in progress to be correlated to the previous results. Significantly, while other methods are commonly used to characterize nanocrystalline drugs, especially in terms of size and morphology (TEM, HRTEM - at the expenses of sample deterioration), the DFA method here discussed offers a novel, exhaustive (and statistically sound) characterization tool ${ }^{[5,6]}$.

Keywords: drugs, debye function analysis, particle size
MS9-P4 Understanding packing interactions and physicochemical properties of novel multicomponent crystal forms of azelaic acid-based anti-inflamatory drugs combining X-ray and NMR

M. Teresa Duarte ${ }^{1}$, Inês C.B. Martins ${ }^{1}$, Mariana $\operatorname{Sardo}^{2}$, Luís Mafra $^{2}$

1. Centro de Quimica Estrutural, Instituto Superior Tecnico, Universidade de Lisboa, Av. Rovisco Pais 1, 1049-001 Lisboa, Portugal,

2. CICECO, Universidade de Aveiro, 3010-193 Aveiro, Portugal

email: teresa.duarte@tecnico.ulisboa.pt

In this work we are presenting complementary studies of X-ray single crystal and powder diffraction as well as SSMNR crystallography in the understanding of packing interactions in multicomponent crystal forms. This approach presents a new insight into the understanding of the new physicochemical properties of this new pharmaceutical entities. Our aim is to optimize crystal engineering methods for the structural elucidation of pharmaceutics, following an X-ray and NMR Crystallography approach where NMR techniques sensitive to crystal packing arrangement are used in tandem with diffraction and computer modeling Structure-activity relationship and solid-state analysis, in particular X-ray diffraction and solid-state NMR spectroscopy, are particularly relevant in pharmaceutical industry where the majority of active pharmaceutical ingredients (APIs) occur as solids. [1, 2] Multicomponent crystal forms of APIs (co-crystals, molecular salts, solvates, hydrates and salts) have been extensively studied over the last years. [2,3] These new forms proved to be an efficient method of improving physicochemical properties of drugs without changing the biological activity, resulting in the improvement of important characteristics such as solubility, dissolution rate, stability under variable RH conditions and bioavailability. [2] Azelaic acid (AA) is an antibacterial product used to treat acne and other skin disorders. This API exhibit low solubility and its performance would benefit from a solubility enhancement. [4] We developed new crystalline solid forms structural, chemical and thermal characterizations will be presented.

Acknowledgments The authors acknowledge funding of the projects POCI/QUI/58791/2004, PEst-OE/QUI/UI0100/2013,

PTDC/CTM-BPC/122447/2010,

RECI/QEQ-QIN/0189/2012 and post-doc grant SFRH/BPD/78854/2011 by Fundação para a Ciência e a Tecnologia

References [1] André, V.; Piedade, M. F. M.; Duarte, M. T., CrystEngComm 2012, 14, 5005-5014. [2] Braga, D.; Maini, L.; Sanctis, G.; Rubini, K.; Grepioni, F.; Chierotti, M. R.; Gobetto, R., Chem.Eur.J. 2003, 9, 5538. [3] Martins, I.; Martins, M.; Fernandes, A.; André, V.; Duarte, M. T., CrystEngComm 2013, 15, 8173. [4] Hebert, R. F., Therapeutically improved salts of azelaic acid. U.S. Patent 6,734,210. May 11, 2004. 